\title{
El Acuerdo de Escazú y sus implicaciones para el sector hídrico en México
}

Autor:

Alberto Rojas Rueda Fecha de publicación: 7 de febrero de 2021
Escazú fortalece la obligación del Estado mexicano para generar estrategias y políticas que garanticen el derecho del público de acceder a la

información en torno al agua.

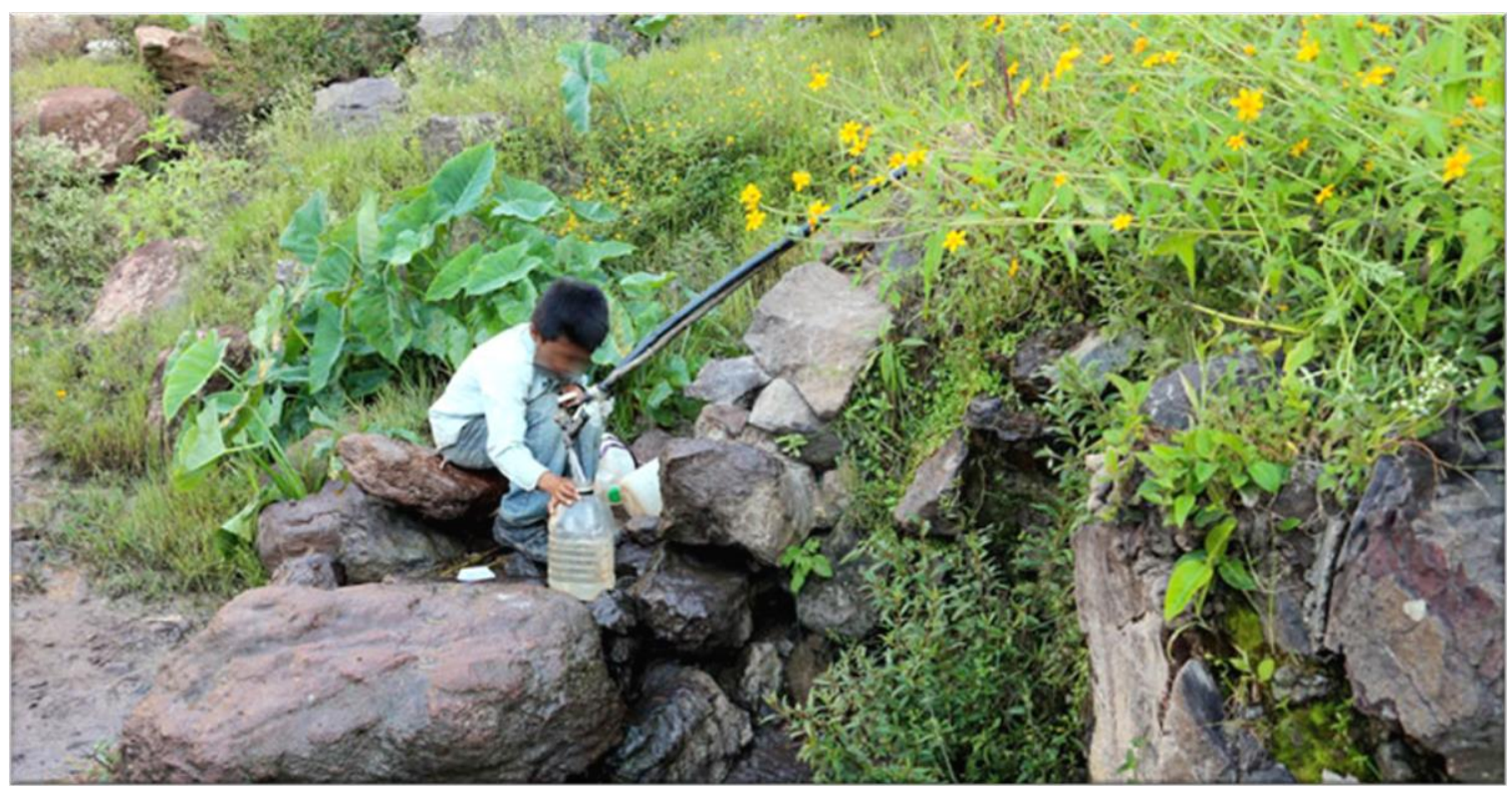

El Acuerdo está conformado por 26 artículos, con enfoque de derechos

humanos, y reconoce los derechos de acceso en materia ambiental

El Acuerdo Regional sobre Acceso a la Información, la Participación Pública y el Acceso a la Justicia en Asuntos Ambientales en América Latina y el Caribe, acordado el 4 de marzo de 2018 en la municipalidad de Escazú, Costa Rica (Naciones Unidas, 2018)-por lo que también se le conoce como Acuerdo de Escazú-, representa el primer esfuerzo multilateral para enfrentar de manera integral asuntos ambientales y de derechos humanos en las regiones latinoamericana y caribeña.

El Acuerdo de Escazú se derivó de la Conferencia de las Naciones Unidas sobre Desarrollo Sostenible, conocida también como Río+20 (celebrada en 2012), en la que 24 países firmaron la Declaración sobre la Aplicación del Principio 10 de la Declaración de Río sobre Medio Ambiente y Desarrollo en América Latina y el Caribe. En 2014 se estableció un pacto para iniciar las negociaciones de un acuerdo regional a través de la creación de un comité de negociación que llevó a cabo nueve reuniones (con la participación de México en la mesa directiva del comité de negociación). 
PERSPECTIVAS IMTA

N ${ }^{\circ}$. 04, 2021

Autor: Alberto Rojas Rueda

DOI: doi.org/10.24850/b-imta-perspectivas-2021-04

La Comisión Económica para América Latina y el Caribe (Cepal) de la Organización de las Naciones Unidas, facilitó el proceso.

México suscribió el documento el 27 de septiembre de 2018. El Senado de la República ratificó el tratado el 5 de noviembre de 2020 (Senado de la República, 2020).

El 22 de enero de 2021, los gobiernos de México, y Argentina, entregaron al secretario general de la ONU (depositario) sus respectivas ratificaciones, por lo que entrará en vigor el próximo 22 de abril de 2021 , Día Internacional de la Tierra', sumando 12 los países que lo han ratificado.

El Acuerdo de Escazú, fundamentado en el principio 10 de la Declaración de Río², tiene carácter jurídicamente vinculante para los países que forman parte del mismo, el cual tiene por objeto luchar contra la desigualdad y la discriminación y garantizar los derechos de todas las personas a un medio ambiente sano y al desarrollo sostenible, dedicando especial atención a las personas y grupos en situación de vulnerabilidad y colocando la igualdad en el centro del desarrollo sostenible (Naciones Unidas, 2018).

El Acuerdo está conformado por 26 artículos, con enfoque de derechos humanos, y reconoce los derechos de acceso en materia ambiental:

Artículo 2, inciso a) por "derechos de acceso" se entiende el derecho de acceso a la información ambiental, el derecho a la participación pública en los procesos de toma de decisiones en asuntos ambientales y el derecho al acceso a la justicia en asuntos ambientales (Naciones Unidas, 2018);

Estos derechos se concentran en cinco temas principales ${ }^{3}$ :

1. La implementación plena y efectiva del acceso a la información ambiental de manera oportuna y adecuada (artículos 5, 6, 12).

2. El reconocimiento del derecho humano a la participación pública en los procesos de toma de decisiones ambientales (artículo 7).

3. El acceso a la justicia en temas ambientales (artículo 8).

4. La protección a defensores de derechos humanos asociados con el ambiente (artículo 9). Escazú es el primer tratado internacional en el mundo que aborda el tema de defensores de derechos humanos. De acuerdo con la organización Global Witness, la región latinoamericana es la de mayor riesgo para los defensores de derechos humanos (Global Witness, 2020). Esta es una de las principales razones por la que este acuerdo regional tiene tanta trascendencia para los derechos humanos.

5. El fortalecimiento de capacidades y la cooperación en materia ambiental (artículos 10 y 11). Este es el pilar del mecanismo que fortalece la cooperación internacional Sur-Sur entre países mediante una estructura institucional de apoyo y diseño de políticas, así como para la toma de decisiones en asuntos ambientales y derechos humanos asociados.

Adicionalmente, establece mecanismos para garantizar el cumplimiento de las obligaciones contraídas por los Estados parte (artículos 13 y 14); una Conferencia de las Partes (artículo 15), que tendrá como secretaria del Acuerdo al Secretariado Ejecutivo a la Cepal (artículo 17), y un Comité de Apoyo a la Aplicación y el Cumplimiento del Acuerdo, como órgano subsidiario de la Conferencia de las Partes (artículo 18). La sede del Secretariado es Santiago de Chile. 
PERSPECTIVAS IMTA

$N^{\circ}$. 04, 2021

Autor: Alberto Rojas Rueda

DOI: doi.org/10.24850/b-imta-perspectivas-2021-04

A nivel global existe otro instrumento regional con características similares, pero de menor alcance (no habla de defensores de derechos humanos), el Convenio sobre el Acceso a la Información, la Participación del Público en la Toma de Decisiones y el Acceso a la Justicia en Materia de Medio Ambiente, adoptado en 1998 en la ciudad de Aarhus, Dinamarca (Unece, 1999).

La relación entre el Acuerdo de Escazú y el sector hídrico deviene de la interpretación amplia que tienen los instrumentos destinados a la protección de derechos humanos que obliga a contemplar los derechos de acceso, aquí descritos, en su relación con las materias que caben dentro de los asuntos ambientales, dentro de los que se encuentran los relacionados con el agua.

En México, los tratados internacionales ratificados por el Senado se consideran parte del bloque de constitucionalidad, por lo que su observancia y aplicación es obligatoria, con un peso similar al de la Constitución Política.

Así, Escazú fortalece la obligación del Estado mexicano para generar estrategias y políticas que garanticen el derecho del público de acceder a la información en torno al agua, así como garantizar su difusión (máxima publicidad); obliga al Estado a reconocer y fortalecer la participación pública en las decisiones que afectan al agua en México, particularmente en la elaboración de los planes y programas; promueve el desarrollo de mecanismos que garanticen el acceso a la justicia hídrica como un elemento de la justicia ambiental; reconoce la obligación del Estado para proteger a defensores de derechos humanos en materia ambiental, entre ellos los asociados con el agua, y, por último, permitirá profundizar en las estrategias para el fortalecimiento de capacidades, así como para la cooperación Sur-Sur en temas hídricos.

En todos estos asuntos, el Instituto Mexicano de Tecnología del Agua (IMTA) ha desarrollado proyectos (indicadores de derechos humanos al agua y saneamiento; transparencia hídrica; justicia hídrica; programas de capacitación). Además, el nuevo arreglo institucional del Instituto se adecua a los cinco pilares de Escazú.

Con el Acuerdo de Escazú, la región contará ahora con el instrumento más avanzado de promoción y protección de los derechos humanos asociados con la protección, conservación y restauración del ambiente, que incluye los temas relacionados con el agua, que, como afirma el secretario general de las Naciones Unidas, Antonio Guterres,

tiene el potencial de catalizar el cambio estructural y dar respuesta a algunos de los principales desafíos de nuestros tiempos. Es un instrumento poderoso para prevenir conflictos, lograr que las decisiones se adopten de manera informada, participativa e inclusiva y mejorar la rendición de cuentas, la transparencia y la buena gobernanza (Naciones Unidas, 2018).

En México, el Acuerdo de Escazú ofrecerá una herramienta para que las personas, en particular quienes pertenecen a grupos en condición de vulnerabilidad, puedan hacer efectivos sus derechos de acceso en torno al agua.

El documento íntegro del Acuerdo se encuentra disponible en: repositorio.cepal.org

Referencias

CEPAL. (03 de 02 de 2021). Principio 10 . Obtenido de www.cepal.org.

Global Witness. (2020). Defender el mañana. Crisis climática y amenazas contra las personas. Uk: Global Witness. 
PERSPECTIVAS IMTA (0)

$N^{\circ}$. 04, 2021

Autor: Alberto Rojas Rueda

DOI: doi.org/10.24850/b-imta-perspectivas-2021-04

Naciones Unidas. (2018). Acuerdo Regional sobre el Acceso a la Información, la Participación Pública y el Acceso a la Justicia en Asuntos Ambientales en América Latina y el caribe. Santiago: CEPAL.

Secretaría de Relaciones Exteriores. (22 de enero de 2021). México hace historia al cumplir los requerimientos para la entrada en vigor del Acuerdo de Escazú. Obtenido de Comunicado conjunto RELACIONES EXTERIORES-MEDIO AMBIENTE: www.gob.mx/sre

Senado de la República. (05 de noviembre de 2020). Avala Senado Acuerdo de Escazú; ayudará a una efectiva protección del ambiente. Obtenido de Coordinación de Comunicación Social: comunicacion.senado.gob.mx Senado de la República. (05 de noviembre de 2020). Gaceta del Senado. Obtenido de Gaceta: LXIV/3PPO-47; www.senado.gob.mx

UNECE. (1999). Convenio sobre el acceso a la información, la participación del público en la toma de decisiones y el acceso a la justicia en materia de medio ambiente. Obtenido de unece.org

${ }^{1}$ El Acuerdo establece que al menos 11 países deben ratificar, aceptar, aprobar o adherirse al mismo para que este entre en vigor 90 días posteriores al depósito del undécimo instrumento (artículo 22, fracción 1,).

${ }^{2}$ El Principio 10 busca asegurar que toda persona tenga acceso a la información, participe en la toma de decisiones y acceda a la justicia en asuntos ambientales, con el fin de garantizar el derecho a un medio ambiente sano y sostenible de las generaciones presentes y futuras" (Cepal, 2021).

${ }^{3}$ Cabe destacar que cualquier país que firme, ratifique, acepte, apruebe o se adhiera a este Acuerdo no podrá formular reserva alguna a su contenido (artículo 23).

${ }^{4}$ Derivado de la entrada en vigor del Acuerdo de Escazú se tendrán que generar indicadores que permitan conocer la línea base de nuestro país en su relación con los derechos de acceso, a fin de verificar el avance en el cumplimiento de sus obligaciones en el marco del mismo. 